\title{
REPOSITÓRIO DATASUS: ORGANIZAÇÃO E RELEVÂNCIA DOS DADOS ABERTOS EM SAÚDE PARA A VIGILÂNCIA EPIDEMIOLÓGICA
}

\author{
Pollianna Marys de Souza e Silva ${ }^{1}$ \\ Universidade Federal da Paraíba \\ pollianna_marys@hotmail.com \\ Marynice Medeiros Matos de Autran ${ }^{2}$ \\ Universidade Federal da Paraíba
}

\begin{abstract}
Resumo
O DataSUS é um repositório de dados, alimentado pelos Sistemas de Informações em Saúde (SIS) do Ministério da Saúde. A Vigilância Epidemiológica (VE) alimenta esses sistemas e estuda o processo saúde-doença. Essa pesquisa objetiva analisar se o repositório DataSUS cumpre com os princípios/critérios preconizados pelo W3C Escritório Brasil para os dados abertos governamentais. Para responder a esse objetivo geral, delimitamos os seguintes objetivos específicos: apresentar a forma de organização do DataSUS por meio de um mapa conceitual; construir o mapa conceitual; demonstrar a relevância do DataSUS para a VE e explicitar a relevância da informação para a VE. Trata-se de uma pesquisa se caracterizada como exploratória e documental. A relevância dos dados abertos governamentais para a VE é um meio de promover uma gestão eficiente nos três entes federativos. Concluise que O DataSUS cumpre com os requisitos preconizados pelo W3C para dados governamentais. Os profissionais da VE possuem em seu poder, através dos dados abertos governamentais, informações capazes de mudar as políticas públicas em saúde.
\end{abstract}

Palavras-chave: DataSUS. Dados Abertos Governamentais. Vigilância Epidemiológica. Mapa Conceitual.

\section{DATASUS REPOSITORY: ORGANIZATION AND RELEVANCE OF OPEN DATA IN HEALTH FOR EPIDEMIOLOGICAL SURVEILLANCE}

\begin{abstract}
DataSUS is a data repository, powered by the Health Information System (SIS) of the Ministry of Health. Epidemiological Surveillance (ES) feeds this system and studies the health-disease process. The research objective is: to analyze whether the DataSUS repository complies with the principles/criteria recommended by the W3C Office Brazil for government Open data. To address this overall objective, we have outlined the following specific objectives: to present the way DataSUS is organized through a concept map; build the concept map; demonstrate the relevance of DataSUS for ES and make explicit the relevance of information for ES. This research is characterized as exploratory and documentary. The relevance of open government data to ES is a means of promoting efficient management across the three federative entities. It concludes that DataSUS complies with the requirements recommended by the $\mathrm{W} 3 \mathrm{C}$ for governmental data. The ES professionals have in their possession, through open government data, information capable of changing public health policies.
\end{abstract}

Keywords: DataSUS. Government Open Data. Epidemiological Surveillance. Conceptual Map.

\footnotetext{
${ }^{1}$ Doutoranda no Programa da de Pós-Graduação em Ciência da Informação - PPGCI/UFPB, Mestre em Serviço Social pela UFPB, Especialista em Análise de Situação em Saúde pelo Instituto de Saúde Pública e Patologia vinculada à Fundação Oswaldo Cruz, Bacharel em Fisioterapia pelo UNIPÊ, Fisioterapeuta Efetiva do Estado da Paraíba - Fisioterapeuta Plantonista da UTI/UCI/Método Canguru da Maternidade Frei Damião e Fisioterapeuta Efetiva do Estado do Rio Grande do Norte - Coordenadora do Núcleo de Educação Permanente do Hospital Regional Monsenhor Antônio Barros.

${ }^{2}$ Professora Associada do Departamento de Ciência da Informação e do PPGCI da Universidade Federal da Paraíba. Doutora em Informação e Comunicação em Plataformas Digitais pela Universidade do Porto em convênio com a Universidade de Aveiro, Mestre em Biblioteconomia pela Dalhousie University - Halifax/Canadá, Especialização em Biblioteconomia em Minas e Energia, pela Universidade de Brasília e Bracharel em Biblioteconomia pela UFPB.
} 


\section{INTRODUÇÃO}

O repositório Institucional DataSUS, pertencente ao Ministério da Saúde (MS), é alimentado por uma rede de sistemas de informações em saúde (SIS), cada um responsável pela coleta de dados de cada área de abrangência do Sistema Único de Saúde (SUS). Este repositório de dados (RD), sediado no Departamento de Informática do SUS, está incumbido de sistematizar, consolidar e veicular as informações produzidas coletivamente, mediante a transferência eletrônica de dados, produzindo os indicadores operacionais e/ou epidemiológicos (RIPSA, 2008).

Uma das áreas de atenção do SUS é a Vigilância Epidemiológica (VE), que estuda o processo saúde-doença através de dados numéricos transformados em informações. Sendo assim, esta área tem como objeto de trabalho a informação. Os profissionais desta área são responsáveis por alimentar os SIS, monitorar os casos registrados nesses sistemas e, através das informações adquiridas nesses sistemas, planejar ações e políticas de saúde pública, contribuindo positivamente com a redução da morbimortalidade e na melhoria das condições de vida da população.

Sabendo que um dos objetivos da VE é melhorar as condições de vida da população, ressaltamos a fala de Freire; Freire (2015): a Ciência da Informação (CI) contribui para a informação se tornar um elemento de inclusão social, trazendo desenvolvimento para as pessoas e seus países. Quando pesquisadores/profissionais dessa ciência organizam documentos para atender a necessidade de um determinado setor da sociedade, devem fazê-lo acreditando que essas informações serão úteis para seus usuários e produzirão benefícios para a população.

Para essa pesquisa, delimitamos o seguinte objetivo geral: analisar se o repositório DataSUS cumpre com os princípios/critérios preconizados pelo W3C Escritório Brasil para os dados abertos governamentais. Para responder a esse objetivo geral, estipulamos os seguintes objetivos específicos: apresentar a forma de organização do DataSUS por meio de um mapa conceitual; construir o mapa conceitual; demonstrar a relevância do DataSUS para a VE e explicitar a relevância da informação para a VE.

De acordo com o W3C Escritório Brasil (2017) os critérios/princípios dos dados abertos governamentais seriam: que estes sejam completos, primários, atuais, acessíveis, compreensíveis por máquina, não discriminatórios, não proprietários e livres de licença. Tais termos serão discutidos no decorrer desse artigo.

A importância da transparência na divulgação dos dados abertos governamentais parte da seguinte premissa: existe uma organização sistemática que predomina na epidemiologia - a 
lógica indutiva. Esta prevê que a partir de um certo número de dados estabelece-se uma proposição mais geral sobre determinado fenômeno (PEREIRA, 2005). Só assim os gestores em saúde podem criar políticas públicas eficazes e capazes de solucionar os problemas de saúde de uma determinada população. A identificação eficaz de um padrão de ocorrência de doenças/agravos nas populações humanas e dos fatores que as influenciam (determinantes e/ou condicionantes) tem sido reiteradamente definida como o objeto de estudo da VE.

Para consolidação da tomada de decisões em saúde pública, a pesquisa em VE precisa alcançar o seu valor integral. Segundo Bonita; Beaglehole; Kjellström (2010) existe um atraso entre a aquisição dos dados (informações) e a sua utilização pelos gestores - os responsáveis pela tomada de decisões e elaboração de políticas públicas em saúde nas três esferas de governo: municipal, estadual e federal.

\section{A RELEVÂNCIA DA INFORMAÇÃO PARA A VE}

Conforme Freire; Freire (2015, p. 115) "[...] vivemos em uma sociedade em que a informação tem sua relevância [...], nessa sociedade as tecnologias digitais possibilitam aos usuários grande mobilidade na busca de informação [...]”. Assim, os SIS, de forma sistemática e descentralizada, contribuem para o acesso livre à informação, permitindo que os usuários (pacientes, gestores e profissionais de saúde) tenham acesso democrático ao conteúdo que desejam. "Pode, portanto, tornar-se um instrumento relevante para auxiliar o planejamento da saúde, definir prioridades de intervenção, além de permitir que seja avaliado o impacto das intervenções" (BRASIL, 2006, p. 7).

A demanda crescente de informações em saúde impõe a criação de mecanismos que acelerem a consolidação desses sistemas, como a coleta adequada, o processamento prático, a análise precisa e a transmissão rápida dessas informações (MORAES; BORDIN, 1994, 2002 apud RITTER; ROSA, 2013). Esses quatro processos, ocorrendo de forma organizada, operacionalizam os serviços de saúde e o planejamento adequado. Partindo desse pressuposto, a utilização efetiva dos SIS (BRASIL, 2007), permite a realização do diagnóstico da ocorrência de um evento na população, fornecendo, inclusive, subsídios para a origem das causas do desenvolvimento das doenças/agravos e indicar riscos aos quais as pessoas estão sujeitas, contribuindo assim para a identificação da realidade epidemiológica de determinada área geográfica.

Tendo a VE a responsabilidade por estudar os padrões de ocorrência de doenças em populações humanas e os fatores determinantes desses padrões (LILIENFELD; LILIENFELD, 
1980) e que estudar, ainda, o processo saúde-doença, analisando a distribuição e os fatores determinantes dos agravos/enfermidades com suas particularidades geográficas (ROUQUAYROL, 1999), para que ocorra uma gestão eficiente nessa área, se faz necessário que os dados abertos governamentais em saúde estejam de acordo com o que preconiza a Secretaria de Logística e Tecnologia da Informação (SLTI) do Ministério do Planejamento Orçamento e Gestão (MPOG):

[...] publicação e disseminação de dados e informações públicas na Web, seguindo alguns critérios que possibilitam sua reutilização e o desenvolvimento de aplicativos por toda a sociedade. A maior parte dos dados e informações geradas ou mantidas pelo governo é pública. Disponibilizar dados na Web não é uma prática recente no governo, porém com uma política de dados abertos, o governo sinaliza que pretende padronizar e alavancar a disseminação de dados públicos por todos os órgãos. (SILVA; CARVALHO, 2018, p. 01).

A VE determina as medidas de prevenção e controle mais indicadas; avalia quais serão as estratégias a serem adotadas (impactos causados) para diminuir e/ou controlar a ocorrência da doença em análise, através do uso dos SIS. As informações obtidas desses sistemas possibilitam a realização de uma análise da situação de saúde de uma população, em uma determinada região. Através da Figura 1 podemos verificar como ocorre o fluxo desses dados.

Figura 1: Fluxograma de Dados para a VE.

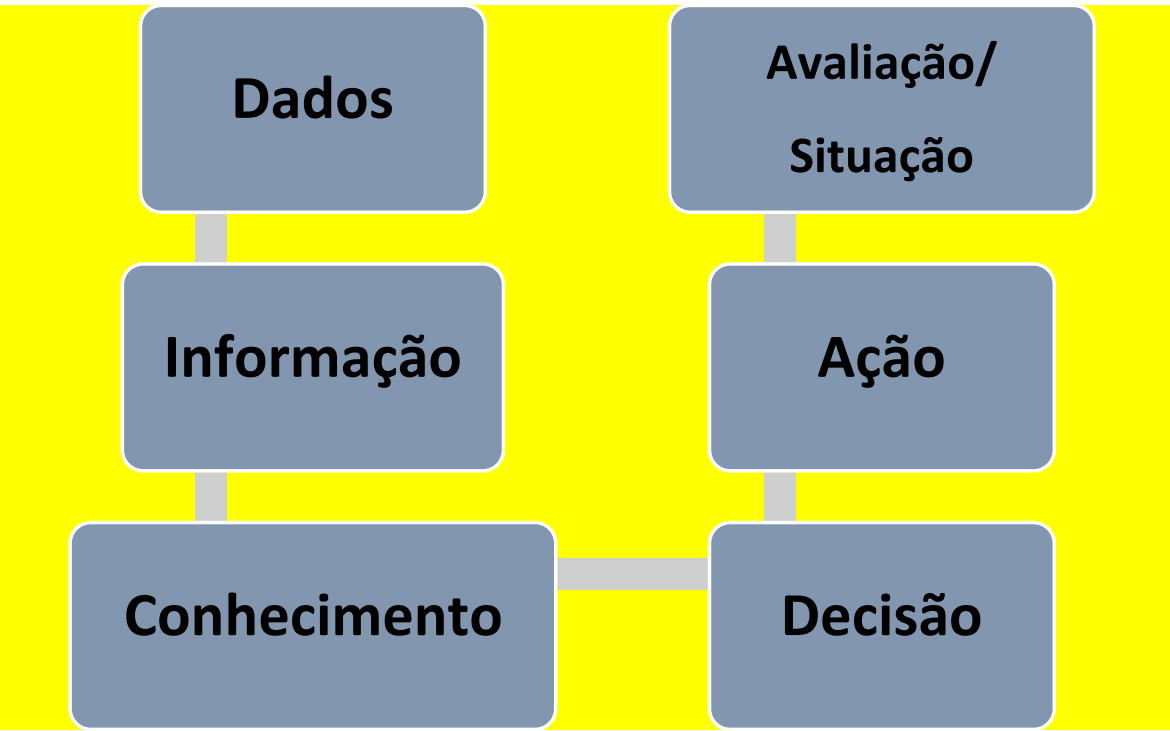

Fonte: Elaborado pelas Autoras, 2019.

\section{PERCURSO METODOLÓGICO}

Esta pesquisa caracteriza-se como exploratória e documental. O repositório DataSUS foi explorado pelas autoras de forma a conseguir concretizar o objetivo proposto. 
A pesquisa documental permite a investigação de determinada problemática (organização do DataSUS) de forma indireta por meio dos documentos que foram produzidos pelos técnicos do MS responsáveis pelo repositório. Estudar documentos implica fazê-lo a partir do ponto de vista de quem os produziu, isso requer cuidado e perícia por parte do pesquisador para não comprometer a validade do seu estudo (CALADO; FERREIRA, 2004)

Para a análise dos dados, as autoras inicialmente tiveram que compreender/estudar a forma que o RD pesquisado é "alimentado" e a distribuição dos dados oriundos dos sistemas dentro do DataSUS.

Em seguida, os sistemas foram distribuídos conforme sua contribuição para cada área que pode ser pesquisada no RD em questão. A partir dessa análise foi construído o Mapa Conceitual do DataSUS utilizando o Cmaps Tools (exposto e discutido nos resultados e discussão).

\section{RESULTADOS E DISCUSSÃO}

Em relação aos dados, e mais especificamente, aos dados abertos governamentais, segundo Pires (2015, p. 11), tais dados devem garantir que duas ou mais bases de dados vindas de fontes diferentes possam ser combinadas sem grandes empecilhos técnicos, evitando "que o governo seja um grande armazém de bases de dados fechadas, ou seja, que não servem senão para a consulta humana, inúteis para aplicação em sistemas maiores e complexos [...]”.

Os indicadores do processo saúde/doença estão distribuídos no DataSUS em seis subconjuntos temáticos: demográficos, socioeconômicos, mortalidade, morbidade e fatores de risco, recursos e cobertura. A produção dos dados brutos utilizados no cálculo dos estudos da VE é fornecida em planilha eletrônica padronizada, preparada pelo DataSUS ou obtida diretamente das bases de dados dos SIS em saúde no Brasil (RIPSA, 2008).

Segundo Ferraz (2009) a informação é fundamental para a democratização da saúde e o aprimoramento de sua gestão. No SUS, a informatização das atividades ocorre através de tecnologias apropriadas, promovendo descentralização das informações e viabilidade do controle social. Conforme ressalta Silva (2010, p. 67-68) tornar públicos os atos de governo é um princípio democrático, que no Brasil aparece expressa no artigo $5^{\circ}$ da Constituição. No inciso XXXIII deste artigo, determina-se que:

[...] todos têm direito a receber dos órgãos públicos informações de seu interesse particular, ou de interesse coletivo ou geral, que serão prestadas no prazo da lei, sob pena de responsabilidade, ressalvadas aquelas cujo sigilo seja imprescindível à segurança da sociedade e do Estado. 
Conhecer os passos de cada uma das etapas sequenciadas de um Sistema de Informação é de fundamental relevância para garantir a fidedignidade das bases de dados, mas também a permanência e plena utilização destas (Quadro 1):

Quadro 1: Etapas de um Sistema de Informação.

\begin{tabular}{|c|c|}
\hline COLETA & $\begin{array}{l}\text { Origem e registros dos dados; } \\
\text { Ordenamentos dos documentos; } \\
\text { Controle da quantidade e do conteúdo; } \\
\text { Transmissão. }\end{array}$ \\
\hline PROCESSAMENTO & $\begin{array}{l}\text { Recebimento e Controle da (avaliação); } \\
\text { Pedido da Informação Adicional; } \\
\text { Codificação; } \\
\text { Digitação e Crítica; } \\
\text { Classificação e Tabulação; } \\
\text { Controle de Erros e Inconsistências; } \\
\text { Cálculos Básicos. }\end{array}$ \\
\hline $\begin{array}{l}\text { DECISÃO } \\
\text { E } \\
\text { CONTROLE }\end{array}$ & $\begin{array}{l}\text { Análise Preliminar dos Dados } \\
\text { Comparação com Parâmetros } \\
\text { Identificação e Análise das Discrepâncias } \\
\text { Opções de Decisão. }\end{array}$ \\
\hline
\end{tabular}

Fonte: Adaptado de Moraes, 1994.

O sistema SIS é operacionalizado no nível administrativo mais periférico, ou seja, nas unidades básicas de saúde, seguindo a orientação de descentralização do SUS. Caso o município não disponha de computadores em suas unidades, o sistema pode ser acessado nas secretarias municipais e/ou estaduais e nas gerências regionais de saúde. Sendo assim, a entrada de dados nos SIS ocorre através da Ficha Individual de Notificação (FIN), preenchida no momento em que o paciente chega a uma unidade de saúde, devendo ser realizada sempre pelo município notificante, independentemente do local de residência do paciente. Depois, essas fichas são encaminhadas, através do boletim de acompanhamento, para os níveis centrais - para o Estado e para as áreas técnicas no MS - onde o processamento eletrônico é feito (BRASIL, 2011).

A manutenção periódica, atualização e avaliação da base de dados desses sistemas tornam-se fundamentais para o acompanhamento da situação epidemiológica dos agravos/doenças e sua utilização efetiva gera a possibilidade de realização do diagnóstico precoce e dinâmico da ocorrência desta enfermidade na população. Ainda permite ao gestor e profissionais de saúde conhecer o desfecho terapêutico dos casos (cura, abandono e óbito), a incidência das formas clínicas, a proporção de casos novos, a prevalência dos casos e a distribuição geográfica destes (FERREIRA; PORTELA; VASCONCELOS, 2000).

A demanda por informações proveniente da Era da Informação e do Conhecimento, vem ocorrendo, inclusive, na área de informática do SUS. A criação da Home Page do DataSUS, que reúne e articula, num único banco de dados, informações de diferentes sistemas, úteis para 
o planejamento e avaliação das políticas públicas de saúde é alimentado pelos SIS, perfazendo um total de nove grupos, como observamos na Figura 1: os hospitalares, de eventos vitais, os financeiros, de gestão, os sociais, os epidemiológicos, o ambulatorial, o estruturante e o de regulação. Cada grupo recebe informações dos seus SIS pertinentes.

Figura 1: Mapa Conceitual - Alimentação do DataSus

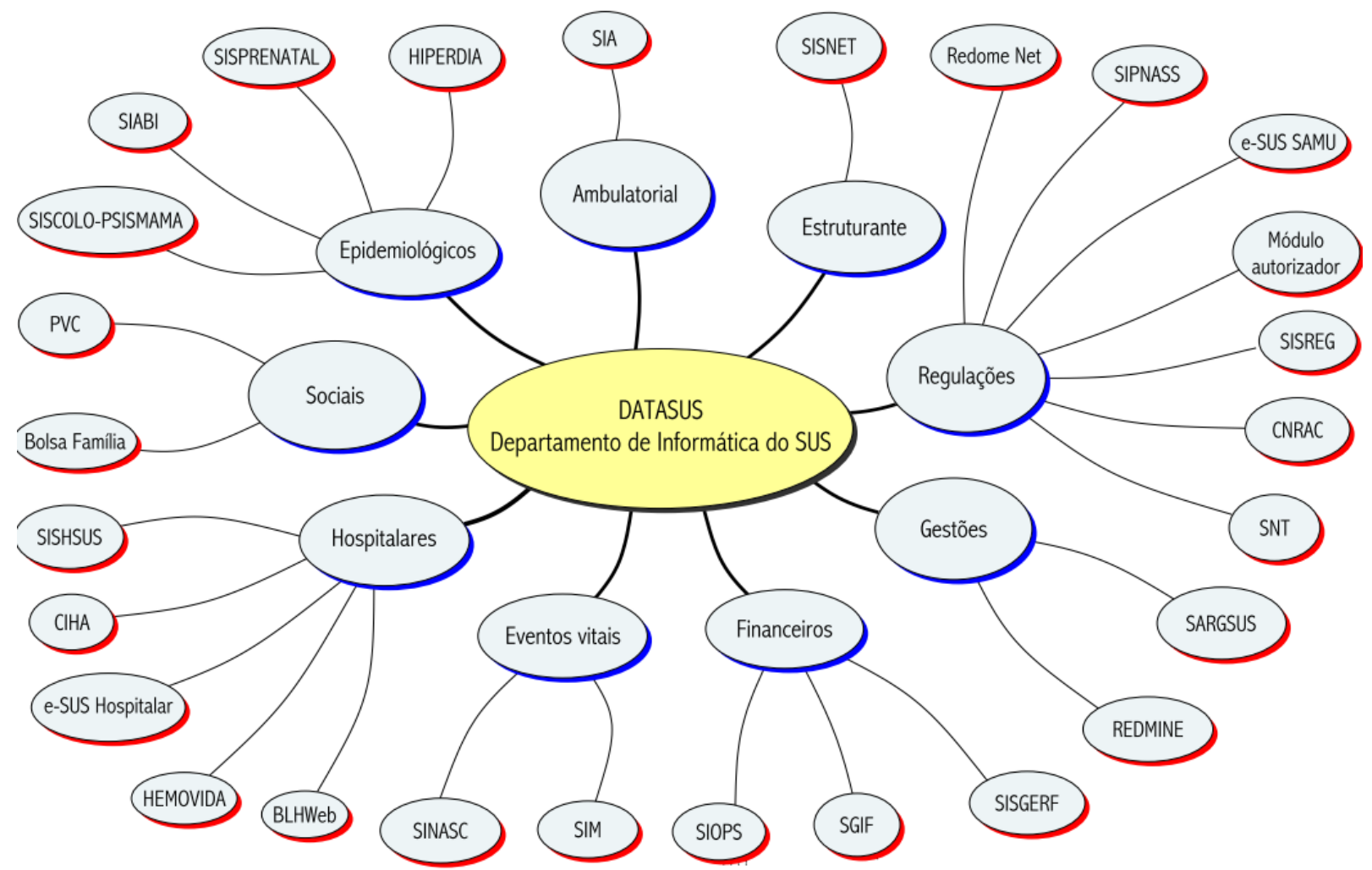

Fonte: Elaborado pelas Autoras, 2019.

Conforme os oitos princípios dos Dados Abertos Governamentais, referidos pelo W3C Escritório Brasil (2017), o DataSUS cumpre com esses princípios, ou seja: possuem dados completos - estão disponíveis e não estão sujeitos a limitações de privacidade, segurança e controle de acesso; são dados primários - apresentados como coletados na fonte, com o maior nível possível de granularidade e sem agregação ou modificação; atuais - os dados são disponibilizados tão rapidamente quanto necessário à preservação do seu valor; acessíveis - os dados são disponibilizados para maior alcance possível de usuários e finalidades; compreensíveis por máquinas - os dados são estruturados de modo a possibilitar processamento automatizado; não discriminatórios - dados disponíveis para todos, sem exigência de requerimento ou cadastro no site; não proprietários - os dados são disponíveis em formato que nenhuma entidade detenha controle exclusivo; e livres de licenças - dados sujeitos a nenhuma restrição de direito autoral, patente, propriedade intelectual ou segredo industrial. 


\section{CONSIDERAÇÕES FINAIS}

Os profissionais da VE possuem em seu poder, através dos dados abertos governamentais, informações capazes de mudar as políticas públicas em saúde da coletividade e melhorar a atuação dos profissionais da atenção básica e hospitalar, baseada na identificação das doenças em determinadas áreas geográficas.

Consideramos que os objetivos propostos foram alcançados, uma vez que mediante a elaboração do Mapa Conceitual demonstramos como o DataSUS está organizado e como ocorre a sua "alimentação" para que sirva como fonte de informação; demonstramos a relevância do DataSUS para a VE; constatamos que o DataSUS cumpre com os princípios/critérios preconizados para os dados abertos governamentais e demonstramos a importância da informação deste RD para a VE.

Apesar da organização do repositório institucional em Saúde no Brasil e da relevância das informações geradas por este, observa-se, de maneira geral, que estas são pouco utilizadas no processo de elaboração das ações de prevenção e tratamento das doenças/agravos notificados, ou seja, as políticas públicas nesta área estão sendo elaboradas sem levar em consideração a relevância da análise dos dados numéricos disponíveis. 


\section{REFERENCIAS}

BONITA, R.; BEAGLEHOLE, R.; KJELLSTRÖM, T. Epidemiologia Básica 2. ed. São Paulo: SANTOS, 2010.

BRASIL. Ministério da Saúde. Secretaria de Vigilância em Saúde. Departamento de Vigilância Epidemiológica. SINAN - Sistema de Informação de Agravos de Notificação (Normas e Rotinas). Brasília: Ministério da Saúde, 2006.

BRASIL. Ministério da Saúde. Secretaria de Vigilância em Saúde. Departamento de Vigilância Epidemiológica. SINAN - Sistema de Informação de Agravos de Notificação (Normas e Rotinas). 2. ed. Brasília: Ministério da Saúde, 2007.

BRASIL. Ministério da Saúde. Secretaria Executiva Departamento de Informática do SUS. Processo de Documentação de Sistemas - PDOC. SINAN Online: manual de operação. Rio de Janeiro: 2011.

CALADO, S. S.; FERREIRA, S. C. R. Análise de Documentos: método de recolha e análise de dados, Metodologia da Educação I. DEFCUL: 2004-2005.

FERRAZ, L. H. V. C. O SUS, o DATASUS e a Informação em Saúde: uma proposta de gestão participativa. 109f. 2009. Dissertação (Mestrado Profissional em Saúde Pública) Escola Nacional de Saúde Pública Sergio Arouca, Rio de Janeiro.

FERREIRA, V. M. B.; PORTELA, M. C.; VASCONCELOS, M. T. L. Fatores Associados à Subnotificação de Pacientes com Aids, no Rio de Janeiro, Revista de Saúde Pública, v. 34, n. 2, p. 170-177, 2000.

FREIRE, G. H. A.; FREIRE, I. M. Introdução à Ciência da Informação. João Pessoa: UFPB, 2015.

LILIENFELD, A. M.; LILIENFELD, D.E. Foundations of Epidemiology. 2. ed. London/New York: Oxford University Press, 1980.

MORAES, I. H. S. Informação em Saúde: da prática fragmentada ao exercício da cidadania. São Paulo: HUCITEC, 1994.

PEREIRA, M. G. Epidemiologia: teoria e prática. Rio de Janeiro: Guanabara Koogan, 2005.

PIRES, M. T. Guia de Dados Abertos. Disponível em:

https://www.slideshare.net/colaborativismo/guia-de-dados-abertos. Acesso em: Julho/2019.

RIPSA. Indicadores Básicos para a Saúde no Brasil: conceitos e aplicações. 2. ed. Brasília: Organização Pan-Americana de Saúde, 2008. 349p.

RITTER, F.; ROSA, R. S. Sistemas de Informação Georreferenciados: como tecnologia para o planejamento das ações em unidades de atenção primária em saúde. In: SCARPARO, H. B. K.; BEDIN, D. M. Gestão em Saúde: experiências de campo e pesquisa com inserção social. Porto Alegre: SULINA, 2013. p. 73-94.

ROUQUAYROL, M. Z. et al. Epidemiologia \& Saúde. 5 ed. Rio de Janeiro: MEDSI, 1999. 
SILVA, C. G.; CARVALHO, F. S. O Negócio de Dados Abertos no Brasil. Disponível em: <file://C:/Users/Windows\%208.1/Desktop/ANÁLISE\%20DE\%20SENTIMENTO/www.dad osabertos.net.html>. Acesso em: 21 out. 2018.

SILVA, D. B. Transparência na Esfera Pública Interconectada. 118f. 2010. Dissertação (Mestrado em Comunicação) - Faculdade Cásper Líbero, São Paulo.

W3C ESCRITÓRIO BRASIL. Dados Abertos Governamentais. Disponível em: http://www.w3c.br/Noticias/CursoDePublicacaoDeDadosEmFormatoAbertoPublicadoPorCar olineBurlePermalinkSemComentarios. Acesso em: 10 jul. 2017. 